\title{
In vitro and in vivo characterization of poractant alfa supplemented with budesonide for safe and effective intratracheal administration
}

Francesca Ricci ${ }^{1}$, Chiara Catozzi ${ }^{1}$, Francesca Ravanetti ${ }^{2}$, Xabier Murgia ${ }^{3}$, Francesco D’Aló ${ }^{1}$, Natalia Macchidani ${ }^{1}$, Elisa Sgarbi ${ }^{1}$, Valentina Di Lallo ${ }^{1}$, Federica Saccani ${ }^{1}$, Marisa Pertile ${ }^{1}$, Antonio Cacchioli ${ }^{2}$, Silvia Catinella ${ }^{1}$, Gino Villetti ${ }^{1}$, Maurizio Civelli ${ }^{1}$, Francesco Amadei ${ }^{1}$, Fabio Franco Stellari ${ }^{1}$, Barbara Pioselli ${ }^{1}$ and Fabrizio Salomone ${ }^{1}$

BACKGROUND: The intratracheal (IT) administration of budesonide using surfactant as a vehicle has been shown to reduce the incidence of bronchopulmonary dysplasia (BPD) in preterm infants. The objective of this study was to characterize the in vitro characteristics and in vivo safety and efficacy of the extemporaneous combination of budesonide and poractant alfa.

METHODS: The stability, minimum surface tension, and viscosity of the preparation were evaluated by means of high-performance liquid chromatography (HPLC), Wilhelmy balance, and Rheometer, respectively. The safety and efficacy of the IT administration of the mixture were tested in two respiratory distress syndrome (RDS) animal models: twentyseventh day gestational age premature rabbits and surfactantdepleted adult rabbits.

RESULTS: A pre-formulation trial identified a suitable procedure to ensure the homogeneity and stability of the formulation. Wilhelmy Balance tests clarified that budesonide supplementation has no detrimental effect on poractant alfa surface tension activity. The addition of budesonide to poractant alfa did not affect the physiological response to surfactant treatment in both RDS animal models, and was associated to a significant reduction of lung inflammation in surfactant-depleted rabbits.

CONCLUSION: Our in vitro and in vivo analysis suggests that the IT administration of a characterized extemporaneous combination of poractant alfa and budesonide is a safe and efficacious procedure in the context of RDS.

M ajor advances in the treatment of premature birth over the last decades such as the extended use of antenatal steroids and surfactant replacement therapy have significantly improved the survival rates of preterm neonates with respiratory distress syndrome (RDS) $(1,2)$, lowering the limits of viability to the twenty-second week of gestation (3).
Nevertheless, preterm infants born at such low gestational ages often require intensive resuscitation approaches with prolonged periods of mechanical ventilation (MV) and continuous exposure to supplemental oxygen. MV and oxygen therapy, often in combination with previous fetal exposures to inflammation (chorioamnionitis), are welldescribed factors known to trigger pulmonary inflammation (4-6). If inflammation persists over time, it can induce the arrest of lung development and impair the normal lung physiology, yielding a chronic lung disease state termed bronchopulmonary dysplasia (BPD) (7). BPD affects about half of preterm neonates with a birth weight below $1,000 \mathrm{~g}$ (refs $8-10)$. In this patient population, non-invasive respiratory support is often inefficient and infants generally require invasive MV and surfactant replacement. In this scenario, the occurrence of inflammation is hard to avoid, increasing the risk of BPD.

Because of the important role of inflammation in BPD (10), corticosteroids have been used in the perinatal context due to their anti-inflammatory effects (11-17). For instance, postnatal systemic dexamethasone therapy has been shown to reduce the incidence of BPD $(16,17)$. Nevertheless, in spite of the pulmonary benefits, the routine use of systemic corticosteroids in premature infants is now discouraged because of the risk of neurodevelopmental impairment (18). As an alternative delivery method, lung-targeted approaches for corticosteroid therapy have been explored. Direct intratracheal (IT) instillation of budesonide alone has been shown to be ineffective (19), whereas administration of inhaled budesonide by aerosol delivery to neonates poses significant technical challenges, and with the current technology the intrapulmonary aerosol deposition rates are variable and relatively low (20). As a result, the efficacy of inhaled corticosteroids in the context of BPD remains inconclusive $(11,13)$.

Yeh et al. (15) have developed an elegant and efficient method for the pulmonary delivery of budesonide to preterm infants. They suspended a dose of budesonide in a dose of

${ }^{1}$ R\&D Department, Chiesi Farmaceutici, Parma, Italy; ${ }^{2}$ Department of Veterinary Science, University of Parma, Parma, Italy; ${ }^{3}$ Department of Drug Delivery, Helmholtz Institute for Pharmaceutical Research Saarland, Saarbrücken, Germany. Correspondence: Fabrizio Salomone (f.salomone@chiesi.com)

Received 21 April 2017; accepted 18 June 2017; advance online publication 23 August 2017. doi:10.1038/pr.2017.171 
exogenous surfactant used for the treatment of RDS. They hypothesized that the surfactant would act as a vehicle for budesonide, and therefore the distribution and the efficacy of budesonide would be maximized. In a recent trial, Yeh et al. (14) have reported a significantly lower incidence of BPD with no observed immediate or long-term adverse effects. The authors also demonstrated that the performance of the bovine-derived surfactant preparation (Survanta, AbbVie, Chicago, IL, USA, phospholipid concentration $25 \mathrm{mg} / \mathrm{ml}$ ) as well as the stability of budesonide were optimal when both substances were combined (14).

Poractant alfa is one of the most effective clinically available exogenous surfactant preparations (21). Preliminary in vitro studies suggest a good surfactant performance of poractant alfa supplemented with budesonide $(22,23)$. Moreover, the combination of poractant alfa with beclomethasone diproprionate, a glucocorticoid with anti-inflammatory properties, showed an optimal surfactant performance in vivo and a reduction of the acute-phase inflammation, suggesting a good compatibility with corticosteroids $(24,25)$. Nevertheless, the in vivo performance of poractant alfa in combination with budesonide has not yet been investigated.

Therefore, considering the growing interest for extemporaneous combinations of surfactant and corticosteroids, the aim of the present study was to perform a preclinical characterization of poractant alfa supplemented with budesonide in order to provide to the clinicians a detailed information on safety and efficacy of a combination of the two products. For that purpose, we prepared an extemporaneous formulation of clinical relevance combining poractant alfa and budesonide. We hypothesized that this formulation would show an equivalent surface tension reduction function in vitro and in vivo compared with poractant alfa alone, yet maintaining the anti-inflammatory properties of budesonide. In addition, viscosity and 24-h stability of the extemporaneous formulation were evaluated with a rotational rheometer and with high-performance liquid chromatography (HPLC), respectively.

\section{METHODS}

\section{Compounds and Reagents}

Poractant alfa (Curosurf, Chiesi Farmaceutici, Parma, Italy) is a sterile aqueous suspension for pulmonary endotracheal instillation. It is a natural surfactant prepared from porcine lungs, constituted of a complex mixture of phospholipids $(80 \mathrm{mg} / \mathrm{ml}$, mainly dipalmytoylphosphatidylcholine) and $\sim 1 \%$ of specific low-molecular-weight hydrophobic proteins.

Budesonide (Pulmaxan, AstraZeneca, Mölndal, Sweden) is a glucocorticoid supplied as a sterile aqueous suspension in single ampules of $2 \mathrm{ml}(0.25 \mathrm{mg} / \mathrm{ml})$ for inhalation via a nebulizer.

For analytical purposes, budesonide was purchased from Sigma (St Louis, MO) and $\mathrm{NaH}_{2} \mathrm{PO}_{4}, \mathrm{H}_{3} \mathrm{PO}_{4}$, absolute ethanol (etOH), and $\mathrm{CH}_{3} \mathrm{CN}$ gradient grade were obtained from Sigma-Aldrich (Milwaukee, WI).

\section{Extemporaneous Formulation Preparation of Poractant Alfa and Budesonide}

As the dose previewed for in vivo studies consists of a dose of $200 \mathrm{mg} / \mathrm{kg}$ of poractant alfa $(2.5 \mathrm{ml} / \mathrm{kg})$ and $0.25 \mathrm{mg} / \mathrm{kg}$ of budesonide $(1 \mathrm{ml} / \mathrm{kg})$, the extemporaneous formulation of poractant alfa and budesonide was prepared for all testing procedures by simply mixing and gently shaking $(5 \times)$ poractant alfa and budesonide in a vial at the following volumetric ratio: 1:0.4 (final poractant alfa and budesonide concentrations were 57.14 and $0.071 \mathrm{mg} / \mathrm{ml}$, respectively).

\section{Budesonide Stability after Mixing with Poractant Alfa}

The stability of the extemporaneous mixture of poractant alfa and budesonide was assessed at different time intervals $(0-24 \mathrm{~h})$ at room temperature by means of HPLC. An HPLC-UV analysis was carried out using a liquid chromatograph (Thermo Fisher Scientific, Cleveland, MA, USA) equipped with an UV-Vis Detector (UV6000 model, Thermo Fisher Scientific) operating at $240 \mathrm{~nm}$. The chromatograms were analyzed with Chromquest 4.0 software (Thermo Fisher Scientific).

Chromatographic separation was performed on a Hypersil BDS C18, a $150 \times 4.6 \mathrm{~mm}, 5 \mu \mathrm{m}$ column (Thermo Fisher Scientific), at room temperature. The mobile phase was an aqueous solution of $0.02 \mathrm{M} \mathrm{NaH}_{2} \mathrm{PO}_{4} \mathrm{pH} 3.2$ (eluent $\mathrm{A}$ ) and $\mathrm{CH}_{3} \mathrm{CN}$ at a ratio of $68: 32$, with a flow rate of $1.5 \mathrm{ml} / \mathrm{min}$. The injection volume was $50 \mu \mathrm{l}$. The total run time was $22 \mathrm{~min}$.

The standard solution of budesonide was prepared by dissolving $\sim 6 \mathrm{mg}$ working standard into a $100 \mathrm{ml}$ of solvent $\mathrm{H}_{2} \mathrm{O} /$ ethanol $(40 / 60 \mathrm{v} / \mathrm{v})$.

The test solution containing poractant alfa and budesonide was prepared as follows: $1 \mathrm{ml}$ of poractant alfa suspension and $400 \mu \mathrm{l}$ of budesonide suspension, both sampled under stirring, were transferred into a $5 \mathrm{ml}$ volumetric flask (sample solution for stability, budesonide sample concentration: $0.071 \mathrm{mg} / \mathrm{ml}$ ). Then, $600 \mu \mathrm{l}$ of water was added and the solution was diluted with ethanol to a final concentration of $0.02 \mathrm{mg} / \mathrm{ml}$.

\section{Determination of the Viscosity of the Expemporaneous Formulation by Rheometer}

The viscosity of the extemporaneous formulation was investigated using a ARG2 Rheometer (TA Instruments, Trios version, New Castle, DE, USA) equipped with cone-plate geometry (plate diameter $40 \mathrm{~mm}$, angle $0.9939^{\circ}$ ) and applying a flow ramp in the rheometer ranging from 0.001 to $10,001 / \mathrm{s}$ shear rates. Poractant alfa alone $(80 \mathrm{mg} / \mathrm{ml})$ was used as a reference. All test samples were gently turned upside down without shaking before analysis. One milliliter of each test suspension was placed directly onto the rheometer's steel plate, and the geometry was lowered up to the defined gap $(27 \mu \mathrm{m})$. All measurements were performed at $25^{\circ} \mathrm{C}$. The viscosity value was extrapolated from the obtained flow ramp curve by applying the Carreau-Yasuda model (TRIOS software, TA Instruments). Measurements were conducted immediately after the preparation of the extemporaneous mixture and again after a $24-\mathrm{h}$ incubation period at room temperature. Results are shown as the mean \pm SD of three runs for each sample (four samples were prepared).

\section{In Vitro Surface Tension Reduction Function of Poractant Alfa after Addition of Budesonide}

To detect potential changes in surface tension attributable to the addition of budesonide to poractant alfa, the surface tension of poractant alfa alone $(80 \mathrm{mg} / \mathrm{ml})$ was measured and compared with that of the extemporaneous combination of poractant alfa/budesonide $(57.14$ and $0.071 \mathrm{mg} / \mathrm{ml}$ concentrations, respectively). Surface tension measurements were performed with a customized Wilhelmy Surface Balance (KSV, Nima, Biolin Scientific Oy, Espoo, Finland). The device uses a platinum plate connected to a strain gauge that is inserted $1 \mathrm{~mm}$ into the hypophase, consisting of $50 \mathrm{ml}$ of $0.9 \% \mathrm{NaCl}$ in a Teflon through. The test samples $(312 \mu \mathrm{l})$ were added into the hypophase. Recordings were then made during 80 cycles for minimum surface tension ( $\gamma \mathrm{min}$ ) measurements. A surface tension value below $3 \mathrm{mN} / \mathrm{m}$ was accepted as optimal surfactant function. All the measurements were performed at $37^{\circ} \mathrm{C}$. The results obtained 


\section{Articles $\mid$ Ricci et al.}

were the mean $\pm S D$ of two runs for each sample (two samples were prepared).

\section{In Vivo Surface Tension Reduction Function of Poractant Alfa after Addition of Budesonide in Preterm Rabbits}

Pregnant New Zealand White rabbits were supplied by Charles River (Domaine des Oncins, Saint-Germain-Nuelles, France) and housed until the twenty-seventh day of gestation under standard conditions, according to the current procedures for animal housing and handling. All experimental procedures involving animal research performed in this work were approved by Italian Ministry of Health (Prot.n ${ }^{\circ}$ 263/2011) and complied with the European and Italian regulations for animal care. The delivery procedure and the postnatal handling of the fetuses have been described elsewhere (26). In total, 27 fetuses from three litters were allocated within four different groups: (i) control group, untreated animals $(n=6)$; (ii) budesonide group, animals received $0.25 \mathrm{mg} / \mathrm{kg}$ of budesonide $(n=6)$; (iii) poractant alfa group, animals received $200 \mathrm{mg} / \mathrm{kg}$ of poractant alfa $(n=7)$; (iv) poractant alfa/budesonide group, animals received 0.25 and $200 \mathrm{mg} / \mathrm{kg}$ of budesonide and poractant alfa combined in an extemporaneous mixture that contained $57.14 \mathrm{mg} / \mathrm{ml}$ of poractant alfa and $0.071 \mathrm{mg} / \mathrm{ml}$ of budesonide $(n=8)$. Fetuses, paralyzed with pancuronium bromide $(0.06 \mathrm{mg}$, intraperitoneal), were ventilated in parallel using a servoventilator (Siemens 900 C, Erlangen, Germany) with a standardized sequence of varying peak insufflation pressures with $100 \%$ oxygen, as previously described (26).

\section{In vivo Efficacy of Surfactant after Addition of Budesonide}

The experiments were carried out in 6- to 7-week-old New Zealand white adult rabbits with a body weight between 1.5 and $2.5 \mathrm{~kg}$. Animal sedation and surgical procedures have been recently described by Ricci et al. (27).

Rabbits, in supine position, were intubated and stabilized on MV (Acutronic Fabian HFO, Acutronic Medical, Hirzel, Switzerland) with the following settings: $\mathrm{FiO}_{2}=100 \%$, flow $=10 \mathrm{l} / \mathrm{min}$, respiratory rate $=40$ breaths $/ \mathrm{min}$, positive end-expiratory pressure $=3 \mathrm{~cm} \mathrm{H}_{2} \mathrm{O}$, $V_{\mathrm{T}}$ targeted to $7 \mathrm{ml} / \mathrm{kg}$ (with peak inspiratory pressure not higher than $23 \mathrm{cmH}_{2} \mathrm{O}$ ), and inspiratory time of $0.5 \mathrm{~s}$. A flow sensor was connected to the endotracheal tube to continuously monitor lung mechanics. All animals underwent repeated bronchoalveolar lavages (BALs) to achieve surfactant depletion as described by Ricci et al. (27). Following surfactant depletion, the animals were randomized to one of the following four study groups: (i) control group, untreated animals $(n=6)$; (ii) budesonide group, animals received $0.25 \mathrm{mg} / \mathrm{kg}$ of budesonide $(n=6)$; (iii) poractant alfa group, animals received $200 \mathrm{mg} / \mathrm{kg}$ of poractant alfa $(n=6)$; and (iv) poractant alfa/ budesonide group, animals received 0.25 and $200 \mathrm{mg} / \mathrm{kg}$ of budesonide and poractant alfa mixed in an extemporaneous mixture that contained $57.14 \mathrm{mg} / \mathrm{ml}$ of poractant alfa and $0.071 \mathrm{mg} / \mathrm{ml}$ of budesonide $(n=6)$. All animals were maintained in MV for $300 \mathrm{~min}$.

Arterial $\mathrm{pH}$, blood gases, dynamic compliance $\left(C_{\text {dyn }}\right)$, and $V_{\mathrm{T}}$ were measured right after intubation and stabilization (baseline), after inducing surfactant depletion by repeated BALs, and after the stabilization period that followed the insult to confirm the respiratory failure (15ST). These parameters were also measured 5, 15 , and $30 \mathrm{~min}$ after treatment, and thereafter every 30 min until the end of the observational period $(300 \mathrm{~min})$. After the observational period, the animals were killed by exsanguination. The lung was fixed for histological analysis.

\section{Histological Analysis}

A standard sampling of the cranial right lung was fixed in $10 \%$ neutral buffered formalin. Lung samples were dehydrated in graded alcohol solutions, xylene clarified, paraffin infiltrated by means of an automatic processor (ATP 700 Tissue Processor, Histo-line Laboratories, Pantigliate, Italy), and embedded with the dorsal surface of the slice down (EG 1160, Leica Biosystems, Mannheim, Germany). Five-micrometer-thick serial sections were obtained using a rotary microtome (Slee Cut 6062, Slee Medical, Mainz, Germany). Slides were further deparaffinized, rehydrated in descending grades of ethanol, and stained with hematoxylin and eosin (Sigma). Images of the samples were acquired with a digital slide scanner (NanoZoomer S60, Hamamazu Photonics, Hamamatsu City, Japan). Ten random nonoverlapping regions of interest were acquired at $\times 5$ magnification for each slide. Lung injury was estimated by using a semiquantitative scoring system performed by an investigator blinded to the study design. Alveolar and interstitial lung inflammation, alveolar and interstitial hemorrhage, edema, and atelectasis were each scored on a $0-4$ point scale $(28,29)$. Each field was scored as 0 if the item was absent or normal, as 1 if the item was present in $25 \%$ of the field, as 2 if it was present in $50 \%$ of the field, as 3 if it was present in $75 \%$ of the field, and as 4 if the item was apparent throughout the whole field. The alveolar and interstitial scores were put together for both lung inflammation and hemorrhage. The total injury score was calculated as a sum of these scores. For each group the average score of the regions of interest is reported.

\section{Statistical Analysis}

Unless otherwise stated, all the data are presented as mean \pm SEM. The raw data of the in vivo studies were analyzed and compared by repeated measures of two-way ANOVA as a function of group and time, followed by Tukey's $t$ post hoc test. The data of the histological score were analyzed by non-parametric Kruskal-Wallis test. Statistical analysis was performed using GraphPad Software (La Jolla, CA, USA) version 6.0 (Prism, CA).

\section{RESULTS}

\section{Budesonide Stability after Mixing with Poractant Alfa}

The stability of the budesonide in the extemporaneous mixture composed of $1 \mathrm{ml}$ of poractant alfa suspension $(80 \mathrm{mg} / \mathrm{ml})$ and $400 \mu \mathrm{l}$ of budesonide $(0.25 \mathrm{mg} / \mathrm{ml})$ was tested immediately after preparation $\left(t_{0}\right)$ and, thereafter, the budesonide content was checked again 3, 6, 9.5, and $24 \mathrm{~h}$ after preparation at room temperature. The chemical stability of budesonide dispersed within poractant alfa over $24 \mathrm{~h}$ was demonstrated using HPLC-UV analysis (Table 1). No significant difference in the content of budesonide was observed during the $24-\mathrm{h}$ incubation at room temperature.

\section{Viscosity of the Extemporaneous Formulation}

The viscosity of poractant alfa $(80 \mathrm{mg} / \mathrm{ml})$ was compared with that of poractant alfa supplemented with budesonide (final poractant alfa and budesonide concentrations were 57.14 and $0.071 \mathrm{mg} / \mathrm{ml}$, respectively) by means of a rheometer. The viscosity of the suspensions was determined immediately after preparation at $t_{0}$, and was determined again after a 24-h incubation at room temperature. The Carreau-Yasuda model was fit to the flow-ramp curves in order to achieve the infinite

Table 1. Stability over time of budesonide within the poractant alfa +budesonide extemporaneous mixture

\begin{tabular}{lcc}
\hline Check-point $(\mathrm{h})$ & Budesonide $(\mu \mathrm{g} / \mathrm{ml})$ & $\begin{array}{c}\text { Budesonide \% } \\
\text { residual vs. initial }\end{array}$ \\
\hline Initial & 18.0 & 100.0 \\
3 & 18.4 & 101.7 \\
6 & 18.2 & 100.6 \\
9.5 & 18.3 & 101.1 \\
24 & 18.6 & 102.8 \\
\hline
\end{tabular}


rate viscosity values. As it could be expected because of the simple phospholipid dilution effect, the addition of budesonide to poractant alfa induced a slight drop of the viscosity from 5.5 to $3.7 \mathrm{cP}$ (30\%; Figure 1).

The 24-h incubation experiment was designed to investigate whether the viscosity of the extemporaneous suspension would remain stable over time. Neither poractant alfa alone nor poractant alfa supplemented with budesonide showed significant changes in viscosity after incubation.

\section{In Vitro Surface Tension Reduction Function of Poractant Alfa after Addition of Budesonide}

The effect of budesonide supplementation on the in vitro surface tension reducing properties of poractant alfa was examined with a Wilhelmy balance and further compared with poractant alfa alone. In both cases the minimum surface tension remained below $3 \mathrm{mN} / \mathrm{m}$, thus confirming the optimal surfactant action of poractant alfa, with or without budesonide supplementation. The film "refinement", defined as the number of dynamic cycles required to reduce minimum surface tension to a level below $3 \mathrm{mN} / \mathrm{m}$, was $4 \pm 0$ cycles for poractant alfa alone and $5.5 \pm 0.7$ cycles for the poractant alfa +budesonide mixture.

\section{In Vivo Surface Tension Reduction Function of Poractant Alfa after Addition of Budesonide}

The in vivo biological activity of poractant alfa combined with budesonide was evaluated in preterm rabbits with primary surfactant deficiency. No significant difference was found between groups in terms of bodyweight. The animal groups treated with poractant alfa or with the poractant alfa +budesonide mixture showed a significantly higher $(P<0.0001) V_{\mathrm{T}}$ compared with untreated animals (Control group) and with animals treated with IT budesonide alone (no surfactant) at 5, 10, 15, and $30 \mathrm{~min}$ (Figure 2). Moreover, the pulmonary improvement achieved after instilling the poractant alfa+budesonide mixture was at the same level as the one observed following instillation of a clinical dose of poractant alfa. On the other hand, budesonide alone could not revert the severe RDS of the preterm rabbits as evidenced by the low $V_{\mathrm{T}}$, at the same level of the untreated controls.

In order to further investigate the in vivo efficacy of poractant alfa in combination with budesonide in terms of gas exchange, lung mechanics, and histological outcomes, we conducted an additional study in adult surfactant-depleted rabbits. Neither the bodyweight of the animals (control group: $1.72 \pm 0.08 \mathrm{~kg}$; poractant alfa group: $1.73 \pm 0.01 \mathrm{~kg}$; poractant alfa+budesonide: $1.7 \pm 0.1 \mathrm{~kg}$; budesonide: $1.7 \pm 0.04 \mathrm{~kg}$ ) nor the number of BALs required to induce surfactant depletion differed significantly between the groups. Moreover, all animals had similar gas exchange and ventilation parameters at baseline as well as $15 \mathrm{~min}$ after induction of surfactant deficiency (15ST, Figure 3). The BALs produced an abrupt decrease in $\mathrm{PaO}_{2}$.

The $\mathrm{PaO}_{2}$ values rapidly increased in all surfactant-treated animals (Figure 3a). Both poractant alfa/budesonide and

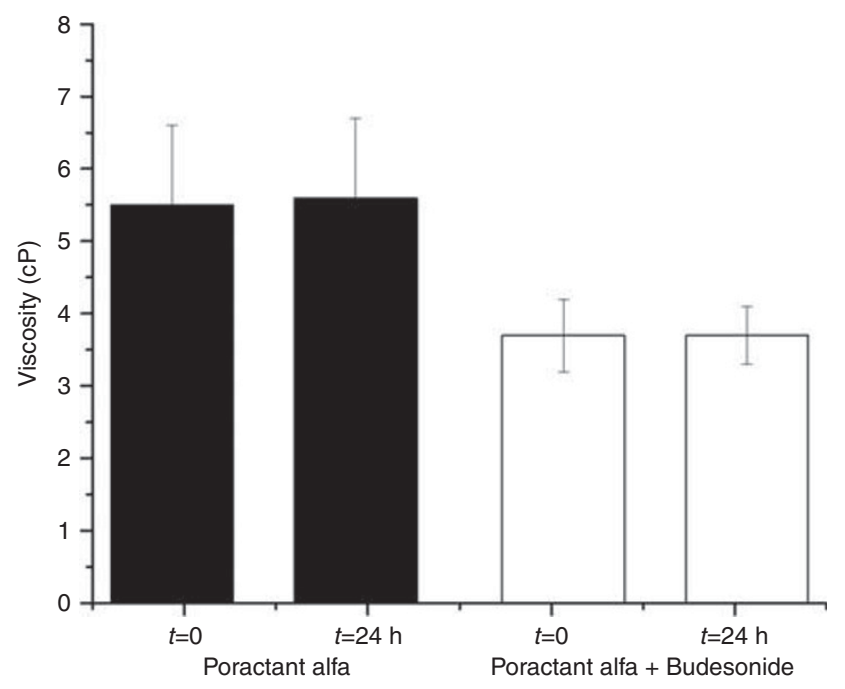

Figure 1. The viscosity of poractant alfa (black bars) was compared with that of the extemporaneous mixture of poractant alfa+budesonide (white bars) right after preparation $(t=0)$ and after a 24-h incubation $(t=24 \mathrm{~h})$. Mean \pm SD are shown.

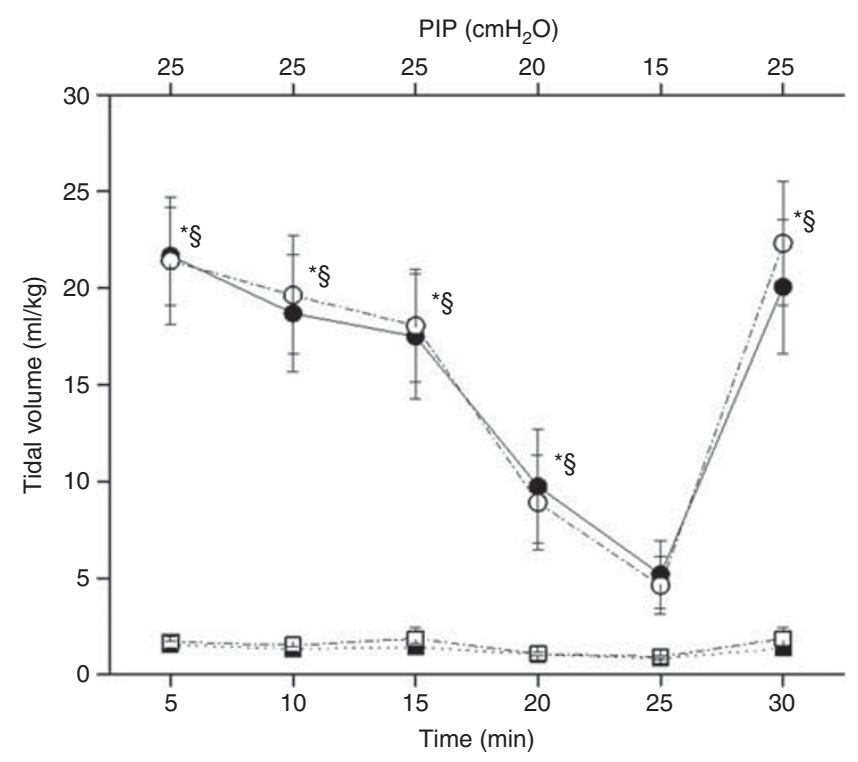

Figure 2. Tidal volumes of preterm rabbits with severe surfactant deficiency treated with poractant alfa ( $200 \mathrm{mg} / \mathrm{kg}$, solid circles), with poractant alfa+budesonide (200 and $0.25 \mathrm{mg} / \mathrm{kg}$, respectively; white circles), and with budesonide only $(0.25 \mathrm{mg} / \mathrm{kg}$, white squares). Untreated animals served as controls (solid squares). Mean \pm SEM are shown. * represents poractant alfa group vs. control and budesonide groups $(P<0.01)$, and ${ }^{\S}$ represents poractant alfa+budesonide group vs. control and budesonide groups $(P<0.01)$.

poractant alfa-only groups had equivalent mean $\mathrm{PaO}_{2}$ values during the whole experimental period, and remained significantly higher in comparison with the mean $\mathrm{PaO}_{2}$ values of the budesonide group and the untreated control group. The oxygenation values of untreated controls and budesonide-treated animals remained below $200 \mathrm{~mm} \mathrm{Hg}$, even though the $\mathrm{FiO}_{2}$ was set at $100 \%$. 


\section{Articles $\mid$ Ricci et al.}
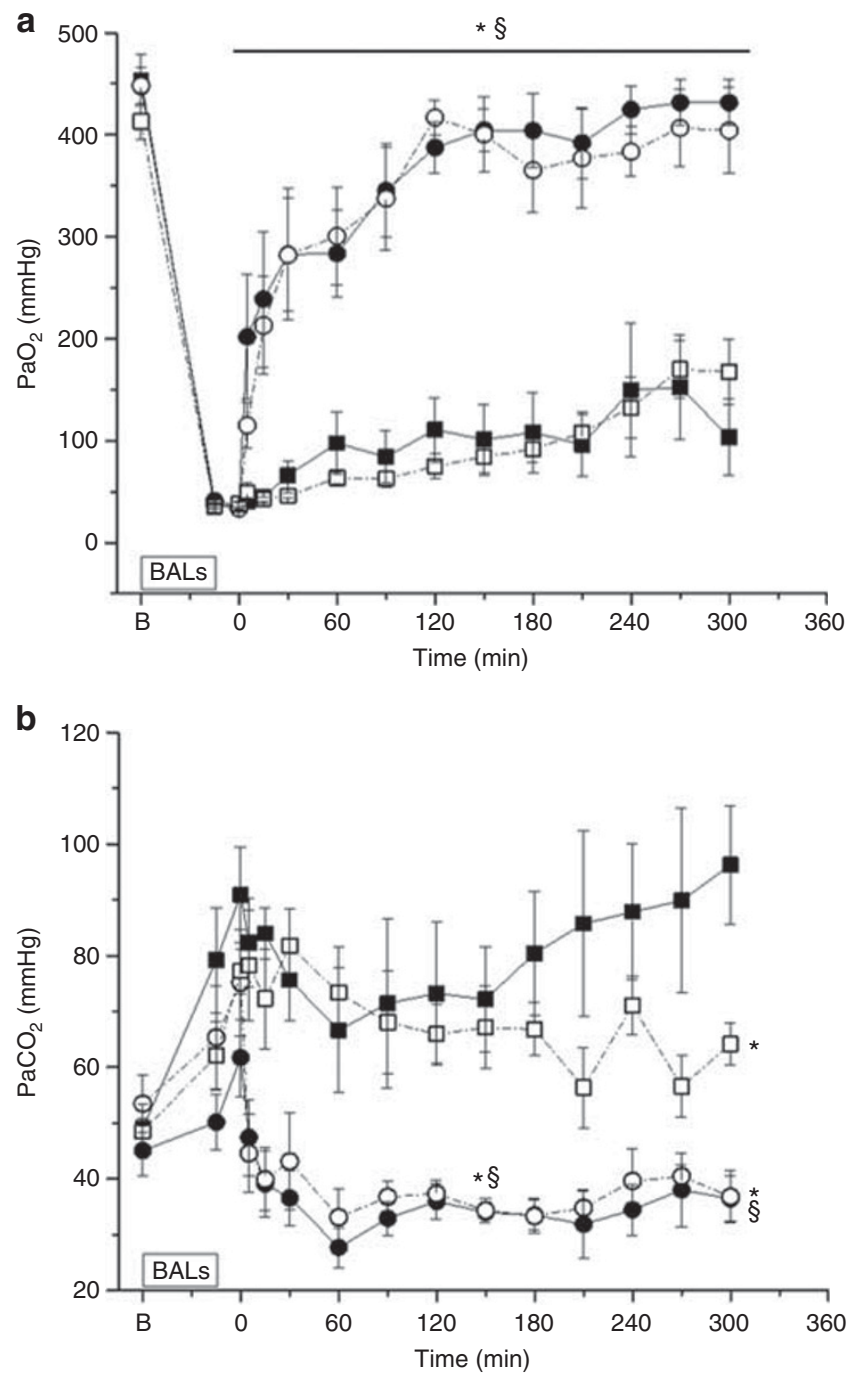

Figure 3. Gas exchange in surfactant-depleted adult rabbits. (a) Mean $\mathrm{PaO}_{2}$ and (b) $\mathrm{PaCO}_{2}$ values of lung-lavaged adult rabbits treated with poractant alfa ( $200 \mathrm{mg} / \mathrm{kg}$, solid circles), with poractant alfa+budesonide (200 and $0.25 \mathrm{mg} / \mathrm{kg}$, respectively; white circles), and with budesonide only $(0.25 \mathrm{mg} / \mathrm{kg}$, white squares). Untreated animals served as controls (solid squares). There were no differences between the experimental groups before the induction of respiratory distress (baseline, B in the $x$ axis). The time point 0 refers to the 15 -min stabilization period established to confirm the respiratory distress induced by repeated BALs. Mean \pm SEM are shown. ${ }^{*}$ vs. control group $(P<0.01)$ and ${ }^{\S}$ vs. budesonide group $(P<0.01)$. BAL, bronchoalveolar lavage.

Surfactant depletion produced hypercapnia and a marked drop in the $\mathrm{pH}$ of all the animals (Supplementary Figure S1 online). Nevertheless, mean $\mathrm{PaCO}_{2}$ and $\mathrm{pH}$ levels returned to normal physiological levels in those animals treated with poractant alfa or poractant alfa+budesonide (Figure $\mathbf{3 b}$ and Supplementary Figure S1). On the other hand, acidosis and hypercapnia persisted in the control group and in the animals treated with budesonide only. It is worth reporting here also that no unexpected events, like liquid reflux or endotracheal tube obstruction, were observed during treatment administration in all groups. At the end of the experimental period, poractant

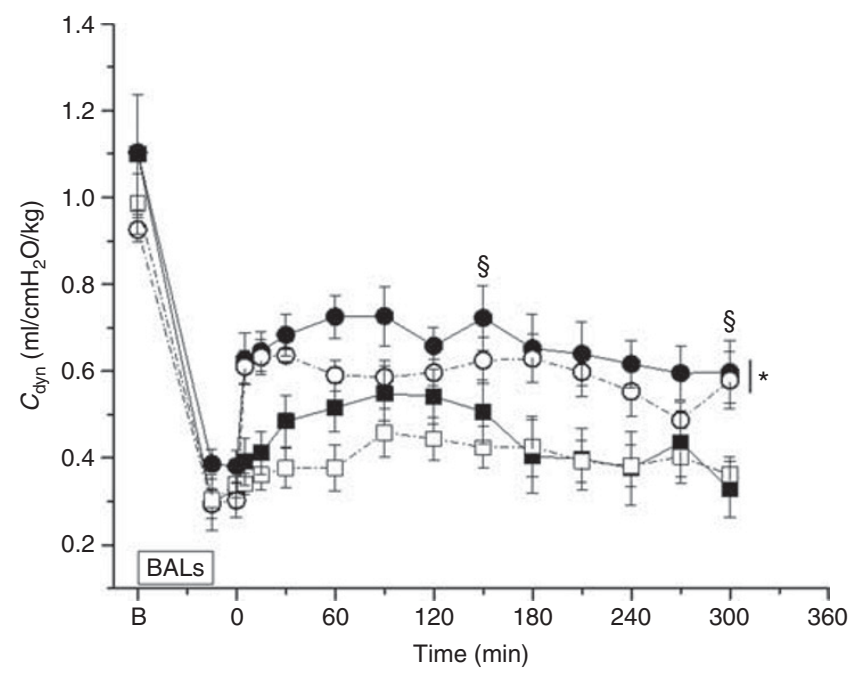

Figure 4. Mean dynamic compliance $\left(C_{\mathrm{dyn}}\right)$ values of lung-lavaged adult rabbits treated with poractant alfa ( $200 \mathrm{mg} / \mathrm{kg}$, solid circles), with poractant alfa+budesonide (200 and $0.25 \mathrm{mg} / \mathrm{kg}$, respectively; white circles), and with budesonide only ( $0.25 \mathrm{mg} / \mathrm{kg}$, white squares).

Untreated animals served as controls (solid squares). There were no differences between the experimental groups before the induction of respiratory distress (baseline, $B$ in the $x$ axis). The time point 0 refers to the 15-min stabilization period established to confirm the respiratory distress induced by repeated BALs. Mean and SEM are shown. ${ }^{*}$ vs. control group $(P<0.01)$ and ${ }^{\S}$ vs. budesonide group $(P<0.01) . B A L$, bronchoalveolar lavage.

alfa/budesonide and poractant alfa groups had significantly better mean $\mathrm{PaCO}_{2}$ and $\mathrm{pH}$ values in comparison with the other groups. Although hypercapnia persisted in the budesonide group, the mean $\mathrm{PaCO}_{2}$ value was significantly lower than that in the untreated controls.

The mean $C_{\mathrm{dyn}}$ values rapidly increased after surfactant treatment in poractant alfa+budesonide and poractant alfa groups (Figure 4). At the end of the experimental period, $C_{\mathrm{dyn}}$ was significantly higher in all surfactant-treated animal groups in comparison with that in the untreated controls. Significant differences were also seen between the poractant alfa group and the budesonide group at the 300 -min time interval. No significant difference was detected between the control and the budesonide-only groups.

\section{Histological Analysis}

The histological examination of the lung parenchyma revealed a mild to marked inflammatory neutrophilic infiltration intermingled with alveolar macrophages, alveolar hemorrhages, and proteinaceous edema. Variably, alveolar walls were thick and atelectatic with multifocal interstitial hemorrhages. These findings, clearly expressing an acute inflammation (Figure 5), affected all the evaluated groups and were more evident in the control group, with varying degrees of severity in treated groups.

Histological examination showed a significant decrease in the overall lung injury score in the poractant alfa+budesonide, poractant alfa, and budesonide groups in comparison with the untreated control group (Table 2). Moreover, significantly 


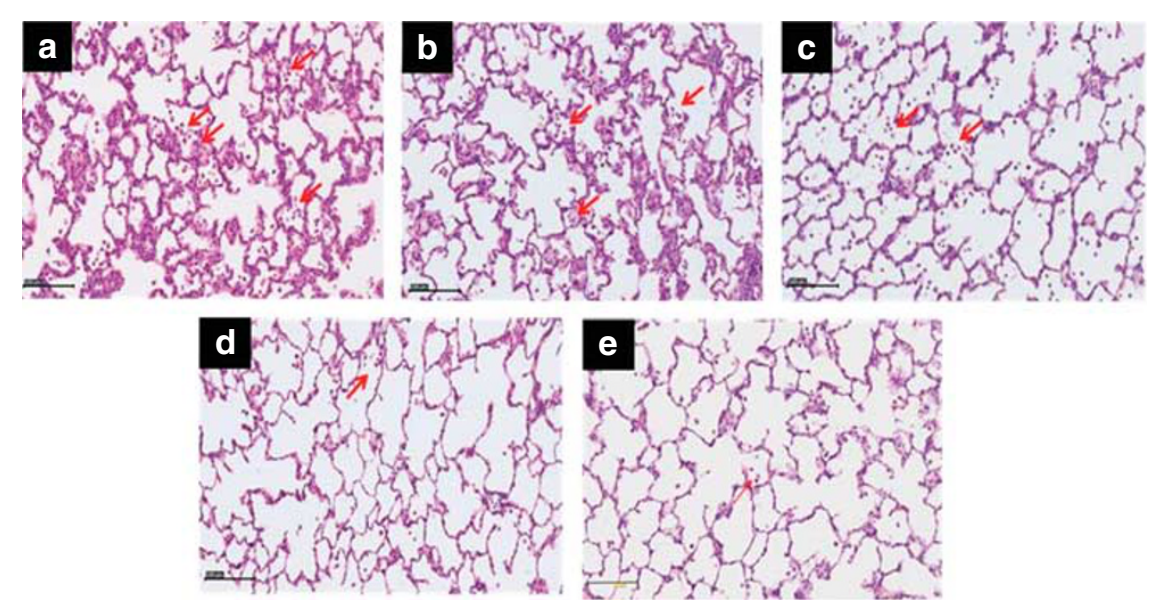

Figure 5. Hematoxylin-eosin staining of lung sections from control (a), poractant alfa (b), budesonide (c), and poractant alfa+budesonide-treated (d), surfactant-depleted adult rabbits. Neutrophilic infiltrates are indicated with a red arrow. The microphotograph of an adult rabbit with normal morphology of the lung parenchyma is shown for comparison (e). Bars (bottom left) $=100 \mu \mathrm{m}$.

Table 2. Results of the histological lung injury score

\begin{tabular}{|c|c|c|c|c|c|}
\hline Group & Inflammation & Hemorrhage & Edema & Atelectasis & Sum score \\
\hline Poractant alfa & $2.61 \pm 0.19^{*}$ & $0.91 \pm 0.18^{*}$ & $1.26 \pm 0.15^{*}$ & $1.30 \pm 0.26^{*}$ & $6.09 \pm 0.58^{*}$ \\
\hline Poractant alfa+budesonide & $1.80 \pm 0.20^{*, \pm, \S}$ & $1.16 \pm 0.12^{*}$ & $0.98 \pm 0.13^{*},+, \S$ & $1.24 \pm 0.16^{*}$ & $5.18 \pm 0.38^{*}$ \\
\hline
\end{tabular}

* Vs. control group, $P<0.01 ;{ }^{\dagger}$ vs. poractant alfa group, $P<0.05 ;{ }^{\ddagger}$ vs. poractant alfa, $P<0.01 ;{ }^{5}$ vs. budesonide group, $P<0.05$.

lower inflammation and edema scores were found for the poractant alfa+budesonide group in comparison to the animal group treated with poractant alfa only.

\section{DISCUSSION}

In the present study we prepared and characterized a clinically relevant extemporaneous formulation of poractant alfa supplemented with budesonide. Our results demonstrate that this formulation could be safely administered for the treatment of RDS and potentially for prevention of BPD in a clinical setting. On the one hand, for the treatment of RDS it is essential that the formulation maintains good surface tension-reducing properties. On the other hand, for the prevention of BPD the budesonide contained within the formulation should be stable and should remain in its active form in order to fully exert its anti-inflammatory properties.

In view of the relevant role played by inflammation in the development of BPD, the use of corticosteroids was rationally suggested. Systemic dexamethasone reduces the incidence of BPD (17). However, a chronic exposure to systemic corticosteroids increases the risk of neurodevelopmental impairment $(18,30)$. Therefore, the local administration of corticosteroids, and in particular of budesonide, has been proposed as an alternative to maximize the lung dose and reduce the side effects of systemic exposure $(11,13,14)$. Yeh et al. (15) developed an elegant approach of pulmonary delivery of budesonide coupled to surfactant replacement therapy. They prepared surfactant/budesonide (beractant/ pulmicort) mixtures (100 and $0.25 \mathrm{mg} / \mathrm{kg}$, respectively) that were delivered to preterm infants with RDS by IT instillation. This approach significantly lowered the incidence of BPD or death $(42 \%)$ in infants treated with the surfactant/budesonide mixture, compared with infants treated with surfactant alone $(66 \%)$.

The aforementioned study prompts us to investigate on an extemporaneous formulation containing a clinical dose of the porcine-derived surfactant poractant alfa $(200 \mathrm{mg} / \mathrm{kg}$ or $2.5 \mathrm{ml} / \mathrm{kg}$ ) and the same budesonide dose as reported by Yeh et al. $(0.25 \mathrm{mg} / \mathrm{kg}$ or $1 \mathrm{ml} / \mathrm{kg})$ in order to provide useful information for the clinical practice on this new treatment approach. We hypothesized that poractant alfa could be a suitable clinical surfactant for this application because similarly to beractant it has a long history of clinical practice and it is an animal-derived cholesterol-free preparation (a critical aspect for budesonide carrier activity according to Zhang et al. (31)). The final concentration of our extemporaneous mixture resulted in a phospholipid concentration of $57.14 \mathrm{mg} / \mathrm{ml}$ and a budesonide concentration of $0.071 \mathrm{mg} / \mathrm{ml}$. Interestingly, the final phospholipid concentration of the extemporaneous poractant alfa and budesonide mixture is still higher than the original phospholipid concentration of beractant $(25 \mathrm{mg} / \mathrm{ml})$ or any other approved natural surfactant preparation (32). Therefore, we hypothesized that the addition of $0.071 \mathrm{mg} / \mathrm{ml}$ of budesonide would not 


\section{Articles | Ricciet al.}

significantly influence the surface properties of poractant alfa. However, investigators were concerned that the sterol budesonide molecule could exert inhibitory effects on surfactant similar to those induced by cholesterol (33-35). The minimum surface tension as measured in vitro for the extemporaneous formulation remained below $3 \mathrm{mN} / \mathrm{m}$, the benchmark set in our in vitro setting for an optimal surface tension-reducing function. This observation is in line with previous studies conducted with natural surfactant mixed with budesonide in which the surfactant action is preserved, provided that the budesonide $\mathrm{wt} \%$ is below $10 \%$ (refs 15,31,35). The extemporaneous formulation used in the present study had a budesonide wt $\%$ of $0.001 \%$. The optimal surfactant function shown in vitro by the poractant alfa+budesonide formulation could be further confirmed in vivo in a preterm rabbit model with severe RDS. Preterm rabbits extracted on the twenty-seventh day of gestation suffer from a severe RDS due to a primary surfactant deficiency and, therefore, this model has been shown to be a good platform to test the efficiency of different surfactant preparations $(26,36)$. Of note, the response curve observed in terms of lung mechanics for the poractant alfa/budesonide group was indistinguishable to the one obtained for the poractant alfaonly group. The mere instillation of budesonide (without surfactant) did not elicit an improvement of lung mechanics, as expected. We could appreciate a slight decrease in the viscosity of the poractant alfa/budesonide extemporaneous formulation in comparison with that of poractant alfa alone. This difference was expected and derives from adding a $40 \%$ volume of budesonide $(0.25 \mathrm{mg} / \mathrm{kg})$ to a clinical dose of poractant alfa $(200 \mathrm{mg} / \mathrm{kg})$. Lower viscosity may actually improve the spreading of the preparation upon instillation, potentially avoiding the risk of transient airway obstructions associated to surfactant installation $(37,38)$.

The chemical stability of budesonide within the extemporaneous poractant alfa+budesonide mixture was also investigated to address if alterations or degradation phenomena of the active form of budesonide could take place over time. The HPLC-UV analysis revealed the stability of budesonide over $24 \mathrm{~h}$ within the extemporaneous poractant alfa+budesonide mixture. A full anti-inflammatory action of budesonide can therefore be expected.

Furthermore, we analyzed the impact of the intratracheal instillation of the surfactant+budesonide mixture on gas exchange and the anti-inflammatory effects of the IT instillation of the poractant alfa+budesonide mixture in surfactant-depleted adult rabbits. Once again, the performance of the extemporaneous mixture was at the same level of the treatment with poractant alfa alone, achieving significant improvements in arterial oxygenation, carbon dioxide removal, and $\mathrm{pH}$. It is noteworthy that no safety issues were identified while dosing the animals with the poractant alfa+budesonide mixture. As seen in the preterm rabbit model, the IT instillation of budesonide alone could not reverse the pulmonary failure induced by the BALs. The histological readouts clearly indicate that the lesions in the control group are indicative of an RDS model (39). Interestingly, however, the animals receiving IT budesonide with or without surfactant obtained the lowest inflammation scores and were assessed with the lowest overall lung injury scores. It is noteworthy to mention that supplementation of poractant alfa with budesonide significantly reduced inflammation and edema in comparison with surfactant treatment alone, indicating a beneficial effect of budesonide supplementation already in the early process of the inflammatory response. Nevertheless, these results must be interpreted, taking into account that the pathophysiology of the pulmonary failure induced by the BALs is different from that observed in neonatal RDS. In addition, the observational period of the study was rather short in order to properly assess the anti-inflammatory effects of budesonide.

In conclusion, we have characterized, in vitro and in vivo, an extemporaneous combination of poractant alfa and budesonide, which shows an optimal surfactant function and an optimal budesonide stability. This formulation is aimed at simultaneously administering a clinical dose of surfactant $(200 \mathrm{mg} / \mathrm{kg})$ and a dose of budesonide $(0.25 \mathrm{mg} / \mathrm{kg})$ known to reduce the incidence of BPD in preterm infants. In the light of our results, this formulation could be potentially appropriate in terms of safety and efficacy to be used in human preterm neonates.

\section{SUPPLEMENTARY MATERIAL}

Supplementary material is linked to the online version of the paper at http://www.nature.com/pr

\section{STATEMENT OF FINANCIAL SUPPORT}

The present study was supported by Chiesi Farmaceutici.

Disclosure: F.R., N.M., E.S., V.D.L., E.S., M.P., S.C., G.V., M.C., F.A., F.F.S., B.P., and F.S. are employees of Chiesi Farmaceutici (Curosurf, poractant alfa, owner). X.M. served as consultants for Chiesi Farmaceutici (Curosurf, poractant alfa, owner) in this study.

\section{REFERENCES}

1. Liggins GC, Howie RN. A controlled trial of antepartum glucocorticoid treatment for prevention of the respiratory distress syndrome in premature infants. Pediatrics 1972;50:515-25.

2. Collaborative European Multicenter Study Group. Surfactant replacement therapy for severe neonatal respiratory distress syndrome: an international randomized clinical trial. Collaborative European Multicenter Study Group. Pediatrics 1988;82:683-91.

3. Guillén Ú, Weiss EM, Munson D, et al. Guidelines for the management of extremely premature deliveries: a systematic review. Pediatrics 2015;136: 343-50.

4. Hillman NH, Moss TJM, Kallapur SG, et al. Brief, large tidal volume ventilation initiates lung injury and a systemic response in fetal sheep. Am J Respir Crit Care Med 2007;176:575-81.

5. Vento M, Moro M, Escrig R, et al. Preterm resuscitation with low oxygen causes less oxidative stress, inflammation, and chronic lung disease. Pediatrics 2009;124:e439-49.

6. Speer CP. Chorioamnionitis, postnatal factors and proinflammatory response in the pathogenetic sequence of bronchopulmonary dysplasia. Neonatology 2009;95:353-61.

7. Hayes D, Feola DJ, Murphy BS, Shook LA, Ballard HO. Pathogenesis of bronchopulmonary dysplasia. Respiration 2010;79:425-36.

8. Jensen EA, Schmidt B. Epidemiology of bronchopulmonary dysplasia. Birth Defects Res Part A Clin Mol Teratol 2014;100:145-57. 
9. Bhandari A, Carroll C, Bhandari V. BPD following preterm birth: a model for chronic lung disease and a substrate for ARDS in childhood. Front Pediatr 2016;4:60.

10. Speer CP. Neonatal respiratory distress syndrome: an inflammatory disease? In. Neonatology 2011;99:316-9.

11. Bassler D, Plavka R, Shinwell ES, et al. Early inhaled budesonide for the prevention of bronchopulmonary dysplasia. N Engl J Med 2015;373:1497-506.

12. Baud O, Maury L, Lebail F, et al. Effect of early low-dose hydrocortisone on survival without bronchopulmonary dysplasia in extremely preterm infants (PREMILOC): a double-blind, placebo-controlled, multicentre, randomised trial. Lancet 2016;387:1827-36.

13. Nakamura T, Yonemoto N, Nakayama M, et al. Early inhaled steroid use in extremely low birthweight infants: a randomised controlled trial. Arch Dis Child Fetal Neonatal Ed 2016;101:F552-6.

14. Yeh TF, Chen CM, Wu SY, et al. Intratracheal administration of budesonide/surfactant to prevent bronchopulmonary dysplasia. Am J Respir Crit Care Med 2016;193:86-95.

15. Yeh TF, Lin HC, Chang $\mathrm{CH}$, et al. Early intratracheal instillation of budesonide using surfactant as a vehicle to prevent chronic lung disease in preterm infants: a pilot study. Pediatrics 2008;121:e1310-8.

16. Yeh TF, Torre JA, Rastogi A, Anyebuno MA, Pildes RS. Early postnatal dexamethasone therapy in premature infants with severe respiratory distress syndrome: a double-blind, controlled study. J Pediatr 1990;117: 273-82.

17. Lin YJ, Yeh TF, Hsieh WS, Chi YC, Lin HC, Lin CH. Prevention of chronic lung disease in preterm infants by early postnatal dexamethasone therapy. Pediatr Pulmonol 1999;27:21-6.

18. Shinwell ES, Karplus M, Reich D, et al. Early postnatal dexamethasone treatment and increased incidence of cerebral palsy. Arch Dis Child Fetal Neonatal Ed 2000;83:F177-81.

19. Lin YJ, Lin HC, Lin CH, Chang SJ, Yeh T. Double-blind controlled trial of endotracheal instillation of budesonide in preterm infants with RDS: a preliminary report. Pediatric Academic Society (PAS), 2000 (http://www. abstract2view.com/pasall/view.php?nu=PASOL_2436).

20. Mazela J, Polin RA. Aerosol delivery to ventilated newborn infants: historical challenges and new directions. Eur. J. Pediatr. 2011;170:433-4.

21. Ramanathan R, Bhatia JJ, Sekar K, Ernst FR. Mortality in preterm infants with respiratory distress syndrome treated with poractant alfa, calfactant or beractant: a retrospective study. J Perinatol 2013;33:119-25.

22. Zuo YY, Zhang H, Wang YE, Neal CR. Differential effects of cholesterol and budesonide on biophysical properties of clinical surfactant. Pediatr Res 2012;71:316-23.

23. Todorov R, Exerowa D, Alexandrova L, et al. Behavior of thin liquid films from aqueous solutions of a pulmonary surfactant in presence of corticosteroids. Colloids Surf A Physicochem Eng Asp 2017;521:105-11.

24. Dani C, Corsini I, Burchielli S, et al. Natural surfactant combined with beclomethasone decreases lung inflammation in the preterm lamb. Respiration 2011;82:369-76.
25. Dani C, Corsini I, Burchielli S, et al. Natural surfactant combined with beclomethasone decreases oxidative lung injury in the preterm lamb. Pediatr Pulmonol 2014;44:1159-67.

26. Ricci F, Murgia X, Razzetti R, Pelizzi N, Salomone F. In vitro and in vivo comparison between poractant alfa and the new generation synthetic surfactant CHF5633. Pediatr Res 2017;81:369-75.

27. Ricci F, Catozzi C, Murgia X, et al. Physiological, biochemical, and biophysical characterization of the lung-lavaged spontaneously-breathing rabbit as a model for respiratory distress syndrome. PLoS ONE 2017;12: e0169190.

28. Mrozek JP, Smith KM, Bing DR, et al. Exogenous surfactant and partial liquid ventilation: physiologic and pathologic effects. Am J Respir Crit Care Med 1997;156:1058-65.

29. Zimmermann AM, Roberts KD, Lampland AL, et al. Improved gas exchange and survival after KL-4 surfactant in newborn pigs with severe acute lung injury. Pediatr Pulmonol 2010;45:782-8.

30. Tam EWY, Chau V, Ferriero DM, et al. Preterm cerebellar growth impairment after postnatal exposure to glucocorticoids. Sci Transl Med 2011;3:1-6.

31. Zhang H, Wang YE, Neal CR, Zuo YY. Differential effects of cholesterol and budesonide on biophysical properties of clinical surfactant. Pediatr Res 2012;71:316-23.

32. Filoche M, Tai C-F, Grotberg JB. Three-dimensional model of surfactant replacement therapy. Proc Natl Acad Sci USA 2015;112:9287-92.

33. Palmer D, Schurch S, Belik J. Effect of budesonide and salbutamol on surfactant properties. J Appl Physiol 2000;89:884-90.

34. López-Rodríguez E, Ospina OL, Echaide M, Taeusch HW, Pérez-Gil J. Exposure to polymers reverses inhibition of pulmonary surfactant by serum, meconium, or cholesterol in the captive bubble surfactometer. Biophys J 2012;103:1451-9.

35. Wang YE, Zhang H, Fan Q, Neal CR, Zuo YY. Biophysical interaction between corticosteroids and natural surfactant preparation: implications for pulmonary drug delivery using surfactant as a carrier. Soft Matter 2012;8:504.

36. Almlén A, Stichtenoth G, Linderholm B, et al. Surfactant proteins B and $\mathrm{C}$ are both necessary for alveolar stability at end expiration in premature rabbits with respiratory distress syndrome. J Appl Physiol 2008;104: 1101-8.

37. Rey-Santano C, Mielgo VE, Andres L, Ruiz-del-Yerro E, Valls-i-Soler A, Murgia X. Acute and sustained effects of aerosolized vs. bolus surfactant therapy in premature lambs with respiratory distress syndrome. Pediatr Res 2013;73:639-46.

38. Rey-Santano C, Mielgo VE, Murgia X, et al. Cerebral and lung effects of a new generation synthetic surfactant with SP-B and SP-C analogs in preterm lambs. Pediatr Pulmonol 52:929-38.

39. Matute-Bello G, Downey G, Moore BB, et al. An official american thoracic society workshop report: features and measurements of experimental acute lung injury in animals. Am J Resp Cell Mol Biol 2011;44:725-38. 